\title{
Glycemic Control and Knowledge among Children and Adolescents with Type 1 Diabetes Mellitus; A Cross-sectional Study
}

\author{
Nosaiba Ahmed Hussein Abdelseed ${ }^{1}$ \\ ${ }^{1}$ Department of Pharmacology, Nile University, Hai El-Gamaa, Al-Ailafoon Road, P.O. Box 11111, \\ Khartoum, Sudan.
}

\section{Article Info \\ Article history: \\ Received:26 November 2020 \\ Revised: 16 February 2021 \\ Accepted: 18 February 2021 \\ Keywords: \\ Glycemic control, Knowledge, Type 1 Diabetes, Children, Adolescents, Sudan. \\ Paper Type : \\ Research Article Corresponding Author: \\ Nosaiba Abdelseed \\ Email: \\ Nosaibahussein@hotmail.com}

\begin{abstract}
Purpose: The aim of this study is to evaluate the long-term glycemic control by glycosylated hemoglobin level (HbAlc) and to assess knowledge about the diabetic regimen. The results of glycemic control in this study were compared with the results of a study conducted in 2018 that targeted almost a similar population.

Study Design: A Cross-Sectional Study.

Subjects and Methods: The study was conducted in October 2012 to December 2012 in a major referral Diabetic Centre. Diabetic children aged 8 to 18 years old who were attending the referral diabetic clinics were included in the study $(n=90)$. Knowledge of diabetes management was evaluated by using a semi-structured questionnaire and HbAlc results were obtained from medical records, available for only 71. Later, these Glycated Hemoglobin HbAlc results were compared with the results of a recent study carried out among Sudanese diabetic children and adolescents in 2018.

Results: Out of 71 diabetic children $78.9 \%$ were of poor glycemic control with mean HbAlc 10.36+2.14. On the other hand, the majority had good knowledge about the diabetic treatment including insulin administration, its storage conditions, and also the effect of exercise on blood glucose. However, almost $18.8 \%$ of studied participants did not know hypoglycemia symptoms, and about $11 \%$ of these patients were unaware of hypoglycemia management.

Conclusion: The study has indicated that a significant percentage of studied diabetic children and adolescents had poor glycemic control. Although children's knowledge about insulin therapy was good, further study must be conducted to investigate factors related to glycemic control among children with type 1 diabetes mellitus (TIDM).
\end{abstract}

\section{Introduction}

Type 1 diabetes is a chronic metabolic disease characterized by destruction of $\beta$-cell that results in lifelong dependence on exogenous insulin (ADA, 2014).The incidence data for Sub-Saharan African countries is limited. Mortality is likely to be high in this part of world(Patterson, 2014).In Sudan the incidence is increasing. During years 2005, 2006, and 2007, it was 2839, 3152 and 3524 respectively (Emad, 2011). The Diabetes Control and Complications trial (DCCT) showed that improved glycemic control decreases long-term diabetes complications in adolescents. In addition, there is evidence that increasing mean HbA1c particularly greater than 9\% significantly increased mortality, more among 
females than males (DCCT/EDIC Research study group, 2016). The American Diabetes Association (ADA) recently recommends an $\mathrm{HbA1c}$ of $<7.5 \%$ in all paediatric age-groups ( $<19$ years of age) but with the goal of achieving the best A1C possible without hypoglycemia (Siminerio, 2014).

A cross sectional study was done among Sudanese children and adolescents with Type 1 Diabetes in 2018, and it was concluded that the majority of participants $(80 \%)$ suffered from high random blood glucose and HbA1c level. Moreover, it was revealed that more than three-quarter (76\%) had poor glycemic control (Taha, 2018). In another published study, it was demonstrated that $86 \%$ of patients had inadequate pre-meal or fasting blood glucose level in their last 6 months. Of the studied patients, it was indicated that $56.6 \%$ were admitted at least once to the hospital in last year, where the main cause of admission was diabetic ketosis (Elrayah, 2005).

There is insufficient information about Type 1 diabetes in Sudan. Therefore, this study aims at describing glycemic control using glycosylated hemoglobin level (HbA1c) as indicator and to evaluate knowledge among diabetic children and adolescents in Khartoum. Ultimately this will help to prevent long term complications.

\section{Methodology and Procedures}

\section{Study Design and Ethical Consideration}

This cross-sectional study was conducted at a large diabetic centre in Khartoum. Ninety of diabetic children age 8 to 18 years who were diagnosed with type I diabetes for at least 6 months during the study period were included. Ethical consideration was obtained from the ethical committee of University of Medical Sciences and Diabetic centre authorities. Permission to collect data was granted from guardians or parents.

\section{Data Collection}

Independent and dependent variables (Demographic data, and knowledge about diabetic regimen e.g., insulin administration, exercise effects, symptoms and treatment of hypoglycemia) were collected by interviewing the patients in the presence of their guardians in waiting room prior to the clinic visit using pre-tested semi-structured questionnaire. $\mathrm{HbA1c}$ levels that were done in the last three months during the study period were obtained from patients' medical records. The results of HbAlc levels were compared with a recent study that targeted one hundred Sudanese diabetic children attending Sudan Childhood Diabetes Centre during study period from October 2017 to March 2018 (Taha, 2018).

\section{Statistical Analysis}

Statistical Package for Social Sciences (SPSS) version 22.0 was used. All categorical variables were expressed in form of table of frequencies and percentages, where continuous variables are reported as mean \pm standard deviation. The chi square was used to determine the association. Multivariate linear regression analysis was performed to find out factors independently associated with glycemic control; p-value less than 0.05 was considered statistically significant. 


\section{Results and Discussion}

\section{Demographic Data}

A total of 90 children with type 1 diabetes were included in this study. Mean age of the study population was $14.08 \pm 2.61$ years, with a range of 8 years to 18 years. Females represent $70 \%$ of the participants. The mean duration of diabetes was $4.96 \pm 3.73$ years. The socio demographic characteristics of studied group are summarized in (Table 1).

Table 1: Socio demographic characteristics of the participants $(n=90)$

\begin{tabular}{|l|l|l|}
\hline Characteristics & Frequency & \\
\hline Sex & & 70 \\
Female & 63 & 30 \\
Male & 27 & \\
\hline Age (Years) & 5 & 5.5 \\
$<10$ & 41 & 45.6 \\
$10-14$ & 44 & 48.9 \\
$>14$ & & \\
\hline Child's education level & 49 & 54.4 \\
Primary school & 37 & 41.1 \\
Secondary school & 4 & 4.4 \\
Some college education & & $\%$ Out of $88^{(1)}$ \\
\hline Duration of Diabetes Mellitus (Years) & 16 & 18.2 \\
$<2$ & 43 & 48.9 \\
2-5 & 29 & 32.9 \\
\hline$>5$ & & $\%$ Out of $89^{(2)}$ \\
\hline Place of origin & 14 & 15.7 \\
Northern Sudan & 6 & 6.7 \\
Eastern Sudan & 38 & 42.7 \\
Central Sudan & 23 & 25.8 \\
Western Sudan & 8 & 8.9 \\
Southern Sudan & 19 & $\%$ Out of $84^{(3)}$ \\
\hline Parent's marital status & 74 & 88 \\
Married & 6 & 7.1 \\
Widowed & 4 & 4.7 \\
Divorced & & $\%$ Out of $82^{(4)}$ \\
\hline Mother's education level & 23.1 \\
Illiterate & & 14.6 \\
Primary & & \\
\hline
\end{tabular}




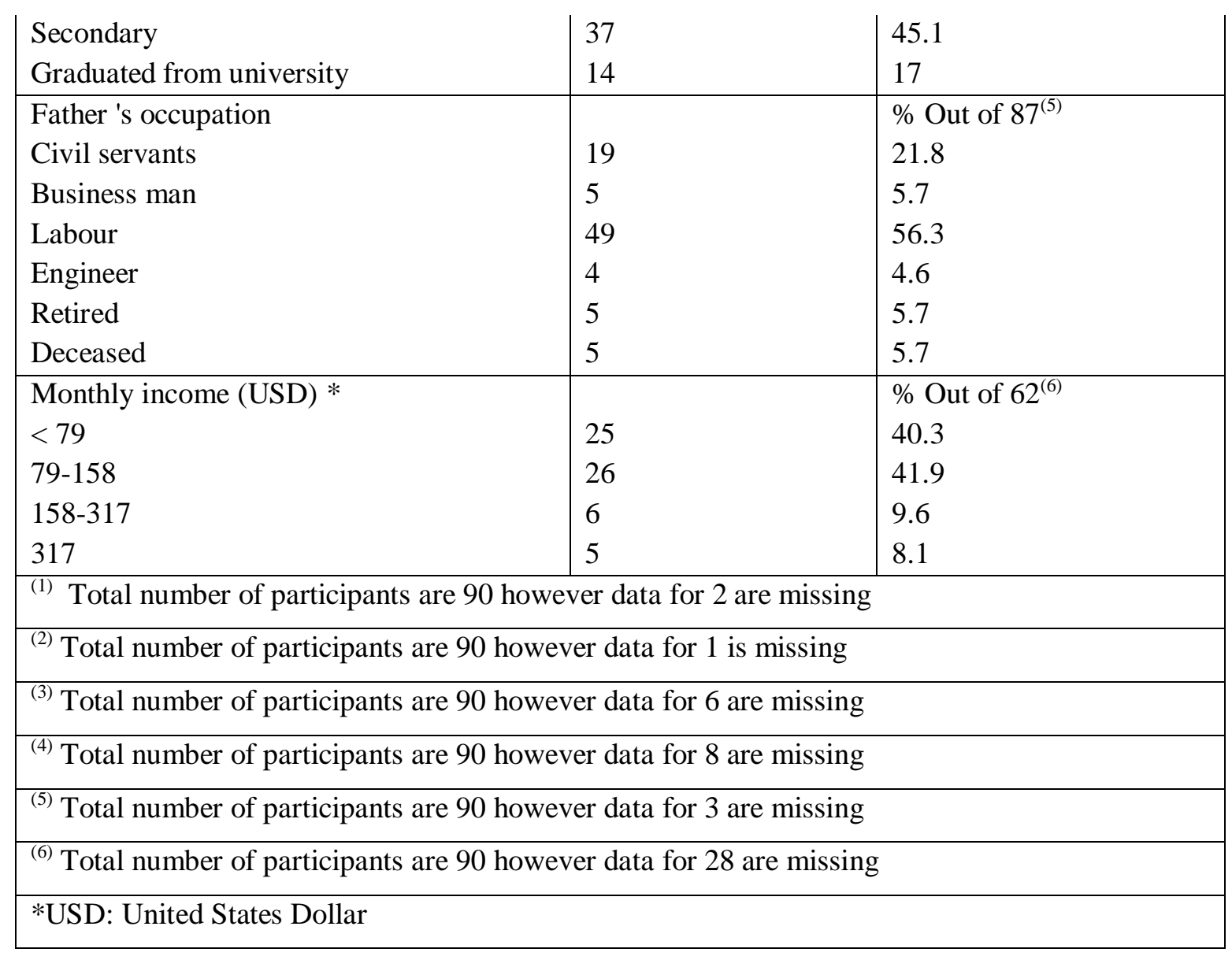

Source: Author

\section{Diabetic control among studied diabetic children and adolescents}

Out of 71 studied participants, $78.9 \%$ were of poor glycemic control when a cut-off HbA1c of $8 \%$ was used (Table 2), with the mean glycemic control HbA1c was $10.36 \pm 2.14 \%$ and a range of $6.2 \%$ to $15 \%$ (Figure1). Short duration of diabetes was significantly associated with lower HbA1c (Table 3). The multivariate regressions show no significant association with $\mathrm{HbA1c}$ and variables tested.

Table 2: Glycemic control of studied diabetic children and adolescents $(n=71)$

\begin{tabular}{|c|c|c|}
\hline Glycemic control & Frequency & \% Out of $71^{*}$ \\
\hline Good control & 15 & 21.1 \\
Poor control & 56 & 78.9 \\
\hline \multicolumn{2}{|c|}{ * Percentages were obtained out of 71( data for19 patients are missing ) } \\
\hline
\end{tabular}




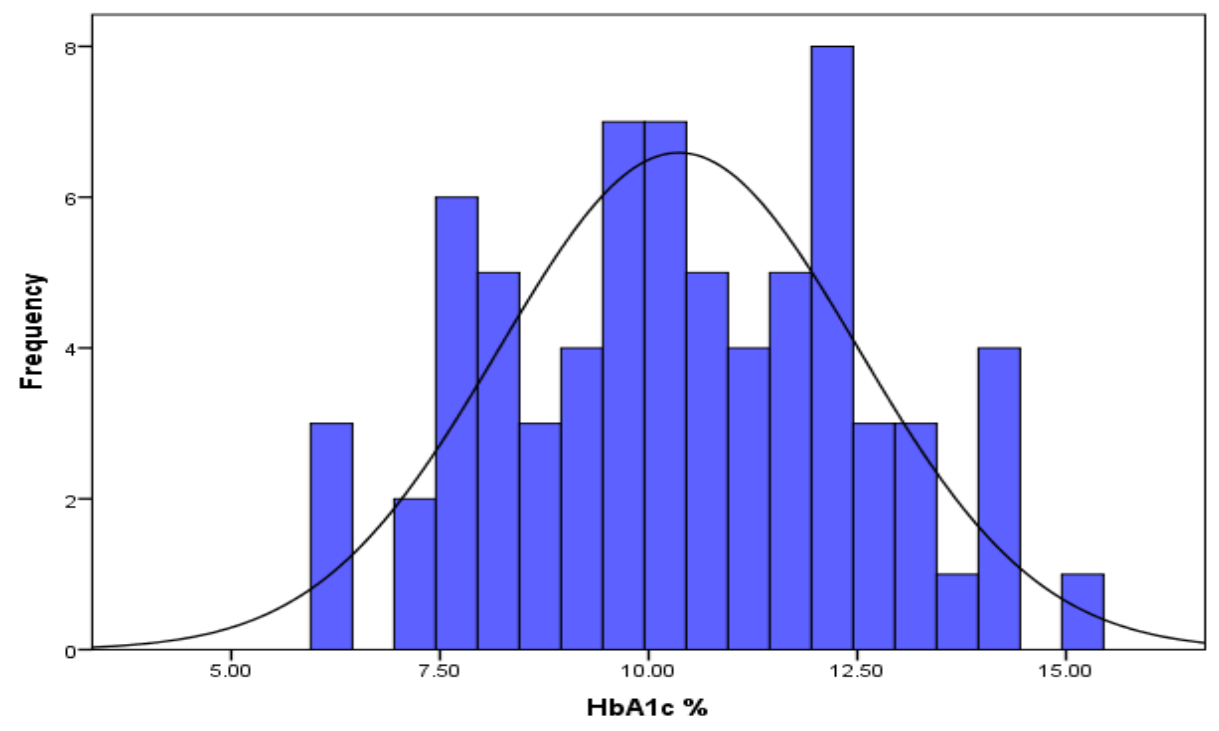

Figure 1: Distribution of HbAlc \% in study population

(Mean 10.36 \pm 2.14 , Median 10.3, Range 6.2-15, kurtosis - 0.753, n 71)

\begin{tabular}{|r|c|c|}
\hline \multicolumn{2}{|l|}{ Table 3: Factor associated with Glycemic control } \\
\hline Characteristic $r$ & Mean HbA1c \pm SD & P value \\
A 10 years & $8.5 \pm 1.44$ & 0.373 \\
10-14 years & $10.45 \pm 2.1$ & \\
$>10$ years & $10.49 \pm 2.2$ & \\
Sex & & \\
Female & $10.3 \pm 2.2$ & 0.217 \\
Males & $10.4 \pm 1.9$ & \\
Diabetes duration & & \\
$<2$ years & $9.8 \pm 2.07$ & \\
2-5 years & $10.54 \pm 2.16$ & \\
& $10.43 \pm 2.2$ & \\
$>5$ years & & \\
Mother's education level & $11.68 \pm 1.57$ & \\
Illiterate & $9.51 \pm 2.09$ & \\
Primary & $10.31 \pm 2.29$ & \\
Secondary & $9.47 \pm 2.18$ & \\
Graduated from university & & \\
\hline P value is statistically significant & $*$ \\
\hline
\end{tabular}


Table 4:Multivariate analysis

\begin{tabular}{|l|l|l|}
\hline Variable & Beta (standard coefficients) & P value \\
\hline Gender & -0.031 & 0.807 \\
\hline Patient age & 0.120 & 0.407 \\
\hline Diabetes duration & 0.088 & 0.511 \\
\hline Child's study level & -0.143 & 0.275 \\
\hline Mother education level & -.0182 & 0.175 \\
\hline
\end{tabular}

Knowledge of study population regarding their diabetic care plan

The vast majority of diabetic children were either having three (64\%) or two insulin injections per day (35\%), about (87\%) of patients inject insulin by themselves and almost (93\%) were able to identify different types of insulin used to control their diabetes. Overwhelming majority of patients reported that they store their insulin in the fridge (89\%), about (10\%) stored in cool places such as clay pot, and only $1 \%$ kept it in room temperature.

On the other hand $(77.8 \%)$ of patients reported that they experienced hypoglycemia episode in which skipping a meal and playing or exercise were the main causes for their hypoglycemia episode. High proportion $(81.2 \%)$ of children recognized the symptoms of low blood glucose as indicated by their response e.g., shakiness, tachycardia, sweating, dizziness, and fatigue. On the contrary about (18.8\%) didn't know the alarming symptoms of low blood glucose. As for hypoglycemia management, high percentage of children (89\%) has good knowledge about hypoglycemia treatment whereas $(11 \%)$ reported inadequate knowledge.

On other side, it was clear that diabetic children and adolescence (92\%) were aware that exercises or physical activities lower their blood glucose level. Regarding home blood glucose monitoring, majority of children and adolescent (89\%) possess glucometer. Sixty percent of these patients showed that are able to use their glucometer, while the remaining proportion in most of these cases their mother responsible for blood glucose measurement.

\section{Discussion}

The present study revealed a result of concern that the prevalence of poor control is $78.9 \%$ with mean HbA1c 10.36 \pm 2.14 . Almost comparable findings reported among one hundred diabetic children in Khartoum by Taha in 2018 in which about $76 \%$ of them had poor glycemic control. This alarming result of HbA1c can have substantial consequences on health outcome (DCCT/EDIC Research study group, 2016). This underlines the importance of lowering level of HbA1c in our population. Studies from developing world have comparable findings. In Cameroon, a study showed similar mean estimates of HbA1c of $10.3 \pm 2.9 \%$ (Niba, 2015). Moreover, close agreement were identified in reports targeted subSaharan African adolescents demonstrating that $67.4 \%$ of participants had poor control and mean HbA1c was $9.2 \pm 2.5 \%$ (Djonou, 2019). Higher estimates documented from Tanzania with mean a HbA1c of $11.1 \pm 2.1 \%$ (Noorani, 2016). In Kenya, a study indicated poor glycemic control with median HbA1c of $11.1 \%$ (Ngwiri, 2015). 
This study showed significant association between glycemic control and diabetes duration. Children with duration of $<2$ year had significantly lower mean HbA1c compared to those with duration $>2$ year (Table 3 ). This could be explained by honeymoon effect which leads to relative good glycemic control due to residual beta cell function after diagnosis and imitation of treatment. Also in comparison with the previous studies that revealed the mother's knowledge about diabetes associated with lower hemoglobin A1c level (Stallwood, 2006) (Tahirovic, 2010), however in our study the mother education level (the school years) was insignificantly associated with glycosylated hemoglobin and this results could be due to the fact that the higher school education level will not ensure diabetes knowledge of these mothers (Table 3).

Regarding multivariate analysis, in the present study no significant predictor of metabolic control was obtained (Table 4). This is in contrary to many studies which demonstrated age, the mother as the primary caregiver, and diabetes duration as independent predictors of glucose control (Noorani, 2016; Niba, 2015). This finding could be due to overall poor glycemic control which may conceal the influence of these factors.

All children and adolescent attending in clinic are offered insulin at no cost. Additionally, the majority of participants had home glucometer as Sudanese paediatric diabetes association offered free glucometer to a large number of diabetic children. However, during data collection period the researcher noted that most patients suffer from cost and availability of BGM strips, but this observation not assessed by current study and require further investigations as previous publications in this context have demonstrated that adherence to BGM is one of predictors for good glycemic control in diabetic children and adolescents(Stallwood, 2006).

Children showed great understanding of insulin storage conditions, as the majority stored their insulin in the right place in fridge and the remaining patients whom home refrigeration unavailable kept insulin in cool places such as clay pots and pocket filled with wet sand; these alternative devices have proven efficient in reducing storage temperature in hot climate of less resourced countries (Ogle, 2016).

The findings of this study reflect that the majority of children have adequate knowledge related to hypoglycemia symptoms but about $18.8 \%$ did not know the alarming symptoms of hypoglycemia. This lack of knowledge about hypoglycemia symptoms can expose the latter group of children to serious consequences including Injury or accident during a hypoglycemic episode, seizures, coma and possibly death, For this reason, it's important to empower patients with knowledge to recognize hypoglycemia symptoms (Clarke, 2009). Additionally, it is important for children and adolescents to know the right intervention to manage mild and moderate hypoglycemia before it gets worse. In this study, $11 \%$ of patients had limited knowledge as signifies by their answers were either: milk, salt, cheese or don't know food/ drink used to treat low blood glucose.

Eventually, this study assessed knowledge about exercise-induced hypoglycemia during and/or after physical activity and children showed great understanding of effect of exercise in reducing the blood glucose level which significance by their answers and this was in good agreement with the published study (Moawad, 2014). 


\section{Conclusion and Suggestion}

The study aimed to evaluate the long-term glycemic control by glycosylated hemoglobin level (HbA1c) and to assess knowledge about the diabetic regimen. The results of glycemic control in this study were compared with the results of a study conducted in 2018 that targeted almost a similar population. The results of the study indicated a significant proportion of studied diabetic children and adolescents had poor glycemic control although overall children knowledge about insulin regimen is good. Therefore, further study should be carried out to investigate the factors related to metabolic control in diabetic children and adolescents, such as adherence to BMG and Diet control.

\section{Abbreviations}

HbA1c: Gycosylated Hemoglobin,

T1DM: Type1Diabetes Mellitus,

DKA: Diabetic Ketoacidosis,

DCCT: Diabetes Control and Complications trial,

ADA: American Diabetes Association,

BMG: Blood Glucose Monitoring.

\section{Acknowledgements}

The author acknowledges paediatric clinics in Jabir Abu Eliz Diabetic Centre, and appreciate the valuable advices provided by prof. Hassan Mohammed Ahmed, Al- Tahir Bashir Osman

\section{Conflict of Interest}

The author declares that they have no competing interests

\section{Funding}

This research received no fund.

\section{References}

American Diabetes Association.(2014). Diabetes through the life span: A position Statement of the American Diabetes Association. Retrieved from https://www.ncbi.nlm.nih.gov/pmc/articles/PMC5865481/.

Clarke, W., Jones, T., Rewers, A., Dunger, D., \&Klingensmith, G.J. (2009) Assessment and management of hypoglycemia in children and adolescents with diabetes.Pediatric Diabetes, 10,(12),134-45. doi: 10.1111/j.1399-5448.2009.00583.x

The Diabetes Control and Complications Trial (DCCT)/Epidemiology of Diabetes Interventions and Complications (EDIC) Study Research Group.(2016). Mortality in type 1 diabetes in the DCCT/EDIC versus the general population.https://care.diabetesjournals.org/content/early/2016/05/14/dc15-2399 
Djonou, C., Tankeu, A.T., Dehayem, M.Y., Tcheutchoua, D.N., Mbanya, J.C.,\&Sobngwi, E.(2019). Glycemic control and correlates in a group of sub Saharan type 1 diabetes adolescents. BMC Res Notes, 12, 50. doi:org/10.1186/s13104-019-4054-1

Elrayah, H., Eltom, M., Bedri, A., Belal, A., Rosling, H., \&Östenson, C.G.( 2005) Economic burden on families of childhood type 1 diabetes in urban Sudan. Diabetes Research and Clinical Practice, 70 (2), 159-65.

Emad, M.A., Ali, Y.H., \&Enan, K.A. (2011). Epidemiology of type 1 diabetes mellitus among children in Sudan: Serological evidence of coxsackievirus infection. Journal of Science and Technology, 12, 64-73.

Moawad, S., Badawy, S. A., Al-saffar, Z. A., Al-Hamdan, N., Awadien, A. M. (2014).Assessment of knowledge among Saudi diabetic children/ adolescent at Riyadh city.American Journal of Nursing Science, 3(1), 5-12. doi: 10.11648/j.ajns.20140301.12

Niba, L., Aulinger, B., MBacham, W., \&Parhofer, K. (2015), Determinants of outcome of children with type 1 diabetes in Cameroon.Horm Res Paediatr, 80, 2-276.

Ngwiri, T., Were, F., Predieri, B., Ngugi, P.,\&Iughetti, L.(2015). Glycemic control in kenyan children and adolescents with type 1 diabetes mellitus. International Journal Endocrinology.doi:org/10.1155/2015/761759

Noorani, M., Ramaiya, K.,\&Manji, K. (2016).Glycaemic control in type 1 diabetes mellitus among children and adolescents in a resource limited setting in Dar es Salaam Tanzania.BMC Endocrine Disorders, 16, 29.doi: 10.1186/s12902-016-0113-y

Ogle, G.D., Abdullah, M., Mason, D., Januszewski, A.S., \&Besançon, S. (2016). Insulin storage in hot climates without refrigeration: Temperature reduction efficacy of clay pots and other techniques. Diabetic Medicine. 33 (11), 1544553.doi: 10.1111/dme.13194

Patterson,C., Guariguata, L., Dahlquist, G., Solte’sz, G., Ogle, G., \&Silink, M.(2014).

Diabetes in the young - a global view and worldwide estimates of numbers of children with type 1 diabetes.Diabetes Research and Clinical Practice, 103, 161-175.

Stallwood, L. (2006). Relationship between caregiver knowledge and socioeconomic factors on glycemic outcomes of young children with diabetes.J Spec PediatrNurs, 11 (3), 158-65.doi: 10.1111/j.1744-6155.2006.00062.x.

Siminerio, L.M., Albanese-O’Neill, A., Chiang, J.L., Hathaway, k., Jackson, C.C....\&Weissberg-Benchell,J. (2014).Care of young children with Diabetes in the child care setting: A position statement of the Americanhttp://main.diabetes.org/dorg/PDFs/Advocacy/Discrimination/ps-care-ofyoung-children-with-diabetes-in-child-care-setting.pdf.

Tahirovic, H., \&Toromanovic, A. (2010) Glycemic control in diabetic children: role of mother's knowledge and socioeconomic status. European Journal pediatrics, 169(8), 961-964.doi: 10.1007/s00431-010-1156-0

Taha, Z., Eltoum, Z., \&Washi, S. (2018). Predictors of glucose control in children and adolescents with type 1 diabetes: Results of a cross-sectional study in Khartoum, Sudan. Open Access Maced J Med Science,6 (11),2035-2039. 